\title{
A REPRESENTATION THEORY FOR A GENERAL CLASS OF CONVOLUTION TRANSFORMS ${ }^{(1)}$
}

\author{
BY \\ I. I. HIRSCHMAN, JR., AND D. V. WIDDER
}

The familiar theorem of S. Bernstein $\left[17\right.$, p. 161] $\left({ }^{2}\right)$ for the representation of a completely monotonic function as a Laplace transform may be considered as providing the theme for the present paper. According to that result a function $f(x)$ has the representation

$$
f(x)=\int_{0}^{\infty} e^{-x t} d \beta(t), \quad \gamma<x<\infty,
$$

where $\beta(t)$ is nondecreasing, if and only if $f(x)$ is completely monotonic,

$$
(-1)^{k} f^{(k)}(x) \geqq 0, \quad k=0,1,2, \cdots,
$$

on the interval $\gamma<x<\infty$. It is natural to inquire whether conditions like (2), but with the $k$ th derivative replaced by a more general differential operator, can be associated with a representation like (1), but with the Laplace transform replaced by a more general integral transform. The operator and transform considered in this connection are those studied by the authors in a previous paper $\left(^{3}\right)$. The notations and results of that paper are here assumed. However, let us state at once, without reference to any previous results, the analogon of Bernstein's theorem referred to above.

Let $a_{1}, a_{2}, \cdots$ be any positive constants such that $\sum_{k=1}^{\infty} 1 / a_{k}^{2}<\infty$, $\sum_{k=1}^{\infty} 1 / a_{k}=\infty$. Define the function $G(t)$ by the equation

$$
G(t)=\frac{1}{2 \pi i} \int_{-i \infty}^{i \infty} \frac{e^{s t} d s}{\prod_{1}^{\infty}\left(1-\frac{s}{a_{k}}\right) e^{s / a_{k}}} .
$$

That is, the bilateral Laplace transform of $G(t)$ is the reciprocal of the entire function

$$
E(s)=\prod_{1}^{\infty}\left(1-\frac{s}{a_{k}}\right) e^{s / a_{k}} .
$$

Presented to the Society, September 10, 1948; received by the editors July 7, 1948.

(1) This research was supported in part by the Office of Naval Research under Contract NR 043-052.

(2) Numbers in brackets refer to the bibliography at the end of the paper.

(3) The inversion of a general class of convolution transforms, Trans. Amer. Math. Soc. vol. 66 (1949) pp. 135-201. The numbering of sections in the present paper is consecutive with the previous one. 
We prove that $f(x)$ has the integral representation

$$
f(x)=\int_{-\infty}^{\infty} G(x-t) d \beta(t), \quad \gamma<x<\infty,
$$

where $\beta(t)$ is nondecreasing, if and only if

$$
\begin{array}{ll}
f(x)=o\left(e^{\alpha x}\right), & x \rightarrow+\infty, \\
f(x) \geqq 0, &
\end{array}
$$

$$
\left(1-\frac{D}{a_{1}}\right)\left(1-\frac{D}{a_{2}}\right) \cdots\left(1-\frac{D}{a_{k}}\right) f(x) \geqq 0, \quad k=1,2,3, \cdots,
$$

on the interval $\gamma<x<\infty$. Here $\alpha$ is the smallest of the constants $a_{k}$. This result reduces, after an exponential change of variable, to Bernstein's theorem when $a_{k}=k$.

We also consider the case in which no restrictions as to sign are placed on the constants $a_{k}$, thus generalizing representations by means of the Stieltjes transform $[17$, p. 365],

$$
f(x)=\int_{0}^{\infty} \frac{d \beta(t)}{x+t}
$$

associated with the conditions

$$
(-1)^{k-1}\left[x^{k} f(x)\right]^{(2 k-1)} \geqq 0,
$$$$
k=1,2, \cdots .
$$

Moreover, necessary and sufficient conditions are obtained for representations (3) in which $\alpha(t)$ is allowed to be in various other classes (of bounded variation, the integral of a function in $L^{p}$, and so on). These conditions are in terms of the linear differential operator (4).

28. The behaviour at infinity of convolution transforms. It is a familiar result that if the Stieltjes transform

$$
f(x)=\int_{0+}^{\infty} \frac{d \alpha(t)}{x+t}
$$

converges, then

$$
\begin{aligned}
f(x) & =o\left(x^{-1}\right) \\
& =o(1)
\end{aligned}
$$$$
\begin{aligned}
& (x \rightarrow 0+), \\
& (x \rightarrow+\infty)
\end{aligned}
$$

Similarly if the Laplace transform

$$
f(x)=\int_{0+}^{\infty} e^{-x t} d \alpha(t)
$$

converges for some values of $x$, and therefore for all sufficiently large $x$, then 


$$
f(x)=o(1)
$$

$(x \rightarrow+\infty)$.

These results are special cases of a general theorem which we shall establish in this section.

THEOREM 28a. If the transform

$$
f(x)=\int_{-\infty}^{\infty} G(x-t) d \alpha(t)
$$

converges for some value of $x$, and if $\alpha_{1}$ and $\alpha_{2}$ are defined as in $\S 9$, then

A. $G(t) \in$ class I implies

$$
\begin{aligned}
f(x) & =o\left(e^{\alpha_{2} x}\right) & & (x \rightarrow+\infty) \\
& =o\left(e^{\alpha_{1} x}\right) & & (x \rightarrow-\infty),
\end{aligned}
$$

B. $G(t) \in$ class II or class III implies

$$
f(x)=o\left(e^{\alpha_{2} x}\right) \quad(x \rightarrow+\infty) .
$$

We shall prove only the first part of conclusion A. The rest follows in a similar manner. Let us recall the asymptotic expansions of Theorem $9 \mathrm{~b}$ for $G(t)$ and $G^{\prime}(t)$ as $t \rightarrow \pm \infty$. We have:

$$
\begin{aligned}
\left(\frac{d}{d t}\right)^{r} G(t) & =\left(\frac{d}{d t}\right)^{r}\left[p(t) e^{\alpha_{1} t}\right]+O\left(e^{\left(\alpha_{1}-\epsilon\right) t}\right) & (t \rightarrow+\infty) \\
& =\left(\frac{d}{d t}\right)^{r}\left[q(t) e^{\alpha_{2} t}\right]+O\left(e^{\left(\alpha_{2}+\epsilon\right) t}\right) & (t \rightarrow-\infty),
\end{aligned}
$$

for $r=0,1$, where $p(t)$ and $q(t)$ are polynomials of degree $\mu_{1}$ and $\mu_{2}$ respectively and $\epsilon$ is some positive constant. As in Definition $9 a, \mu_{1}+1$ is the multiplicity of $\alpha_{1}$ as a zero of $E(s)$, and so on. Choose $x_{0},-\infty<x_{0}<\infty$, and set

$$
A(t)=\int_{t}^{\infty} G\left(x_{0}-u\right) d \alpha(u) .
$$

We have

$$
\begin{aligned}
f(x)= & -\int_{-\infty}^{\infty} \frac{G(x-t)}{G\left(x_{0}-t\right)} d A(t) \\
& =\left[-A(t) G(x-t) / G\left(x_{0}-t\right)\right]_{-\infty}^{\infty}+\int_{-\infty}^{\infty} A(t)\left[G(x-t) / G\left(x_{0}-t\right)\right]^{\prime} d t .
\end{aligned}
$$

Using equations (1) we obtain

$$
\left[-A(t) G(x-t) / G\left(x_{0}-t\right)\right]_{-\infty}^{\infty}=A(-\infty) e^{\alpha_{1}\left(x-x_{0}\right)}=O\left(e^{\alpha_{1} x}\right) \quad(x \rightarrow+\infty) .
$$


Let

$$
\int_{-\infty}^{\infty} A(t)\left[G(x-t) / G\left(x_{0}-t\right)\right]^{\prime} d t=I_{1}+I_{2}
$$

corresponding to the ranges of integration $(-\infty, T)$ and $(T, \infty)$. We have

$$
\begin{aligned}
\left|I_{1}\right| & \leqq \sup _{-\infty<t \leqq T}|A(t)| \int_{-\infty}^{T}\left|\frac{d}{d t}\left[G(x-t) / G\left(x_{0}-t\right)\right]\right| d t, \\
I_{2} & \leqq \sup _{T \leqq t<\infty}|A(t)| \int_{T}^{\infty}\left|\frac{d}{d t}\left[G(x-t) / G\left(x_{0}-t\right)\right]\right| d t .
\end{aligned}
$$

If $x \geqq x_{0}$ then $\left[G(x-t) / G\left(x_{0}-t\right)\right]^{\prime}$ is non-negative for $-\infty<t<\infty$, see [13]. Hence

$$
\begin{aligned}
\left|I_{1}\right| & \leqq \sup _{-\infty<t \leqq T}|A(t)|\left\{\frac{G(x-t)}{G\left(x_{0}-t\right)}-\frac{e^{\alpha_{1} x}}{e^{\alpha_{1} x_{0}}}\right\} & \\
& =O\left(x^{\mu_{1}} e^{\alpha_{1} x}\right) & (x \rightarrow+\infty) .
\end{aligned}
$$

Finally

$$
\begin{aligned}
\left|I_{2}\right| & \leqq \sup _{T \leqq t<\infty}|A(t)|\left\{\frac{e^{\alpha_{2} x}}{e^{\alpha_{2} x_{0}}}-\frac{G(x-t)}{G\left(x_{0}-t\right)}\right\}, \\
& \leqq \sup _{T \leqq t<\infty}|A(t)| e^{-\alpha_{2} x_{0}} e^{\alpha_{2} x} .
\end{aligned}
$$

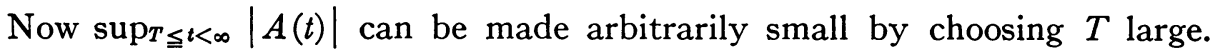
Our theorem follows.

\section{A general representation theorem.}

Definition 29a. Let $\nu_{1}$ be the smallest value of $n$ for which the multiplicity of $\alpha_{1}$ as a zero of $E_{n}(s)$ does not exceed one, and the multiplicity of $\alpha_{2}$ does not exceed one. Let $\nu_{2}$ be the smallest value of $n$ for which neither $\alpha_{1}$ nor $\alpha_{2}$ are zeros of $E(s)$.

Let $G(t)$ be a kernel corresponding to the constants $b,\left\{a_{k}\right\}_{1}^{\infty}$, and let $\left\{P_{n}(D)\right\}_{1}^{\infty}$ be an inversion operator for $G(t)$ corresponding to $\left\{b_{k}\right\}_{1}^{\infty}$. We may associate with $G(t)$ a "normalized" kernel $G^{\sharp}(t)$ corresponding to $0,\left\{a_{k}\right\}_{1}^{\infty}$ and a "normalized" inversion operator $\left\{P_{n}^{\#}(D)\right\}_{1}^{\infty}$ for which $b_{k}=0, k=1,2, \cdots$. The reader will easily convince himself that in the succeeding theorems it is sufficient to consider in the proofs only "normalized" kernels and inversion operators. The theorems will be stated in general but will be proved only for this special case, which however implies, by the simplest transformations, the validity of the theorems in question for the general case. The advantage of this procedure lies in the considerable simplification of the notation. 
TheOREM 29b. Let $f(x) \in C^{\infty}(-\infty<x<\infty)$. If

1a.

$$
G^{\sharp}(t) \in \text { class I, }
$$

2a.

$$
\begin{aligned}
f^{(n)}(x) & =o\left(e^{\alpha_{2} x}\right) & & (x \rightarrow+\infty ; n=0,1,2, \cdots) \\
& =o\left(e^{\alpha_{1} x}\right) & & (x \rightarrow-\infty ; n=0,1,2, \cdots)
\end{aligned}
$$

or if

$1 \mathrm{~b}$.

$$
G^{\sharp}(t) \in \text { class II, }
$$

$2 \mathrm{~b}$.

$$
\begin{aligned}
f^{(n)}(x) & =o\left(e^{\alpha_{2} x}\right) \\
& =o\left(e^{\theta x}\right)
\end{aligned}
$$$$
(x \rightarrow+\infty ; n=0,1,2, \cdots)
$$$$
(x \rightarrow-\infty ; n=0,1,2, \cdots)
$$

for some $\theta<0$, or if

1c.

$$
G^{\#}(t) \in \text { class III, }
$$

2c.

$$
f^{(n)}(x)=o\left(e^{\alpha_{2} x}\right)
$$$$
(x \rightarrow+\infty ; n=0,1,2, \cdots)
$$

then for $m \geqq \nu_{1}, n \geqq \nu_{2}$, and $(-\infty<x<\infty)$

$$
\int_{-\infty}^{\infty} G_{m}^{\#}(x-t) P_{n}^{\#}(D) f(t) d t=\int_{-\infty}^{\infty} G_{n}^{\#}(x-t) P_{m}^{\#}(D) f(t) d t .
$$

We shall prove this theorem under assumptions $1 \mathrm{a}$ and $2 \mathrm{a}$. The other cases are, if anything, simpler.

We assert that if one of the integrals of equation (1) exists then ithe other integral exists and they are equal. The reader may easily verify the identities

$$
\begin{gathered}
(1-D / a) e^{D / a} \phi(t)=-(1 / a) e^{D / a} e^{a t} D e^{-a t} \phi(t), \\
{\left[\phi(x-t) e^{D / a} \psi(t)\right]_{-\infty}^{\infty}=\left[\psi(t) e^{-D / a} \phi(x-t)\right]_{-\infty}^{\infty},} \\
\int_{-\infty}^{\infty} \phi(x-t) e^{D / a} \psi(t) d t=\int_{-\infty}^{\infty} \psi(t) e^{-D / a} \phi(x-t) d t .
\end{gathered}
$$

If we set

$$
I=\int_{-\infty}^{\infty} G_{m}^{\#}(x-t) P_{n}^{\#}(D) f(t) d t,
$$

we find on integrating by parts and using equations (2) that

$$
\begin{aligned}
I= & {\left[\left\{-\frac{1}{a_{n}} e^{-D / a_{n}} e^{a_{n} t} G_{m}^{\#}(x-t)\right\}\left\{e^{-a_{n} t} P_{n-1}^{\#}(D) f(t)\right\}\right]_{-\infty}^{\infty} } \\
& +\int_{-\infty}^{\infty}\left\{\left(1+\frac{D}{a_{n}}\right) e^{-D / a_{n}} G_{m}^{\#}(x-t)\right\} P_{n-1}^{\#}(D) f(t) d t .
\end{aligned}
$$


Using assumption $2 \mathrm{a}$ and the asymptotic estimates of Theorem $9 \mathrm{~b}$ for $G_{m}(t)$ we see that the integrated term vanishes and

$$
I=\int_{-\infty}^{\infty}\left\{\left(1+\frac{D}{a_{n}}\right) e^{-D / a_{n}} G_{m}^{\#}(x-t)\right\} P_{n-1}^{*}(D) f(t) d t .
$$

Repeating this process $n-m$ times we find that

$$
\begin{aligned}
I & =\int_{-\infty}^{\infty}\left\{\prod_{m+1}^{n}\left(1+\frac{D}{a_{k}}\right) e^{-D / a_{k}} G_{m}^{\#}(x-t)\right\} P_{m}^{\dagger}(D) f(t) d t \\
& =\int_{-\infty}^{\infty} G_{n}^{\dagger}(x-t) P_{m}^{\#}(D) f(t) d t .
\end{aligned}
$$

Our proof will now be complete if we can show that for $n \geqq \nu_{2}$ one of the integrals of equation (1) is convergent. By Theorem $9 \mathrm{~b}$ for $n \geqq \nu_{2}$ we have

$$
\begin{aligned}
G_{n}^{\#}(t) & =O\left(e^{\left(\alpha_{1}-\epsilon\right) t}\right) & & (t \rightarrow+\infty), \\
& =O\left(e^{\left(\alpha_{2}+\epsilon\right) t}\right) & & (t \rightarrow-\infty),
\end{aligned}
$$

for some positive constant $\epsilon$. Using assumption $2 \mathrm{a}$ we see that for $n \geqq \nu_{2}$ the integral

$$
\int_{-\infty}^{\infty} G_{n}^{\#}(x-t) P_{m}^{\#}(D) f(t) d t
$$

is absolutely convergent.

THEOREM 29c. Under the hypotheses of Theorem 29b we have

$$
\lim _{n \rightarrow \infty} \int_{-\infty}^{\infty} G_{m}^{\#}(x-t) P_{n}^{\#}(D) f(t) d t=P_{m}^{\#}(D) f(x) .
$$

For $n \geqq \nu_{2}$ we have by Theorem $29 \mathrm{~b}$ that

$$
\begin{aligned}
\int_{-\infty}^{\infty} G_{m}^{\sharp}(x-t) P_{n}^{\#}(D) f(t) d t & =\int_{-\infty}^{\infty} G_{n}^{\#}(x-t) P_{m}^{\sharp}(D) f(t) d t \\
& =\prod_{\nu_{2}+1}^{n}\left(1-\frac{D}{a_{k}}\right) e^{D / a_{k}} \int_{-\infty}^{\infty} G_{\nu_{2}}^{\#}(x-t) P_{m}^{\dagger}(D) f(t) d t .
\end{aligned}
$$

By Theorem 19b we see that

$$
\lim _{n \rightarrow \infty} \prod_{\nu_{2}+1}^{n}\left(1-\frac{D}{a_{k}}\right) e^{D / a_{k}} \int_{-\infty}^{\infty} G_{\nu_{2}}^{\#}(x-t) P_{m}^{\#}(D) f(t) d t=P_{m}^{\sharp}(D) f(x),
$$

from which our theorem follows.

30. An auxiliary lemma. The following elementary result is to be used in conjunction with the theorems of the preceding section. 
LEMMA 30. If

1a. $G^{*}(t) \in$ class $\mathrm{I}$,

2a. $f(x) \in C^{n}(-\infty<x<\infty)$,

3a. $f^{(k)}(x)=o\left(e^{\alpha_{1} x}\right)$

$$
\begin{aligned}
& (x \rightarrow+\infty ; k=0,1, \cdots, n-1) \\
& (x \rightarrow-\infty ; k=0,1, \cdots, n-1)
\end{aligned}
$$$$
=o\left(e^{\alpha_{2} x}\right)
$$

then
A.
$f(x)=g_{1} * g_{2} * \cdots * g_{n} * P_{n}^{*}(D) f(x)$
$(-\infty<x<\infty)$.

If

1b. $G^{t}(t) \in$ class II or III,

2b. $f(x) \in C^{n}\left(\gamma_{c}<x<\infty\right)$,

3b. $f(x)=o\left(e^{\alpha_{2} x}\right)$

$$
(x \rightarrow+\infty ; k=0,1, \cdots, n-1)
$$

then

B. $f(x)=g_{1} * g_{2} * \cdots * g_{n} * P_{n}^{*}(D) f(x) \quad\left(\gamma_{c}<x<\infty\right)$.

Suppose that assumptions $1 \mathrm{a}, 2 \mathrm{a}$, and $3 \mathrm{a}$ are satisfied. Integrating the linear differential equation

$$
\left[\left(1-\frac{D}{a_{n}}\right) e^{D / a_{n}}\right] P_{n-1}^{\#}(D) f(x)=P_{n}^{\#}(D) f(x),
$$

we see that

$$
P_{n-1}^{*}(D) f(x)=-a_{n} e^{a_{n} x-1} \int_{0}^{x-1 / a_{n}} e^{-a_{n} t} P_{n}^{*}(D) f(t) d t+E e^{a_{n} x-1},
$$

where $E$ is a constant which we must determine from assumption 3a. There are two possibilities, $a_{n} \geqq \alpha_{2}$, and $a_{n} \leqq \alpha_{1}$. We shall consider only the first of these, which is typical. By assumption we have

$$
P_{n-1}^{\#}(D) f(x)=o\left(e^{\alpha_{2} x}\right) \quad(x \rightarrow+\infty) .
$$

Allowing $x$ to become positively infinite in (2) we see that if $a_{n} \geqq \alpha_{2}$ equation (2) can hold only if the integral

$$
\int_{0}^{\infty} e^{-a_{n} t} P_{n}^{\#}(D) f(t) d t
$$

converges to the value $E / a_{n}$. Equation (2) may therefore be rewritten as

$$
P_{n-1}^{*}(D) f(x)=g_{n} * P_{n}^{*}(D) f(x) \text {. }
$$


Repeating this argument $n$ times we obtain conclusion A. The proof of conclusion B is entirely similar.

31. Some elementary Tauberian theorems. The results contained in this section may be deduced from well known theorems by an exponential change of variable, see [3]. Proofs are included both for the sake of completeness and also because the demonstrations are, in this form, much simpler than those usually given.

THEOREM 31a. If

1.

2.

3.

$$
\begin{aligned}
f(x) & \in C^{2}(0 \leqq x<\infty), \\
f(x) & =o\left(e^{\alpha x}\right), \quad \alpha>0 \\
f^{\prime \prime}(x) & \geqq O\left(e^{\alpha x}\right)
\end{aligned}
$$$$
\begin{aligned}
& (x \rightarrow+\infty), \\
& (x \rightarrow+\infty),
\end{aligned}
$$

then

$$
f^{\prime}(x)=o\left(e^{\alpha x}\right)
$$$$
(x \rightarrow+\infty) \text {. }
$$

Let $\theta$ be any real constant not equal to zero. The identity,

$$
f^{\prime}(x)=\theta^{-1}[f(x+\theta)-f(x)]+\theta^{-1} \int_{x+\theta}^{x}(x+\theta-t) f^{\prime \prime}(t) d t,
$$

may be verified by integrating by parts. By assumption 3 there exists a nonnegative constant $A$ such that $f^{\prime \prime}(x)>-A e^{\alpha x}(0 \leqq x<\infty)$. We may establish by elementary estimations from equation (1) that

$$
\begin{aligned}
f^{\prime}(x) & \leqq o\left(e^{\alpha x}\right)+\theta A e^{\alpha(x+\theta)} & & (x \rightarrow+\infty ; \theta>0) \\
& \geqq o\left(e^{\alpha x}\right)+\theta A e^{\alpha(x+\theta)} & & (x \rightarrow+\infty ; \theta<0) .
\end{aligned}
$$

Since $\theta$ may be chosen arbitrarily small these equations imply

$$
f^{\prime}(x)=o\left(e^{\alpha x}\right) \quad(x \rightarrow+\infty),
$$

as desired.

TheOREM 31b. Let $Q(D)$ be a linear differential operator of degree $n$ with constant coefficients.

$$
Q(D)=q_{n} D^{n}+q_{n-1} D^{n-1}+\cdots+q_{0}, \quad\left(q_{n} \neq 0\right) .
$$

If

1. $f(x) \in C^{n}(0 \leqq x<\infty)$,

2. $f(x)=o\left(e^{\alpha x}\right), \quad \alpha>0 \quad(x \rightarrow+\infty)$,

3. $\int_{0}^{x} Q(D) f(t) d t \geqq O\left(e^{\alpha x}\right) \quad(x \rightarrow+\infty)$,

then 


$$
f^{(k)}(x)=o\left(e^{\alpha x}\right) \quad(x \rightarrow+\infty ; k=1,2, \cdots, n-2) .
$$

It is sufficient to prove our theorem for $k=1$, because we may then deduce it for $k=2,3, \cdots, n-2$. Suppose that it has been established for $k=1$; we have $f(x), f^{\prime}(x) \in C^{n-1}(0 \leqq x<\infty), f^{\prime}(x)=o\left(e^{\alpha x}\right)$ as $(x \rightarrow+\infty)$, and if $Q^{*}(D)$ $=q_{n} D^{n-1}+q_{n-1} D^{n-2}+\cdots+q_{1}$ then, by assumption 3 ,

$$
\int_{0}^{x} Q^{*}(D) f^{\prime}(t) d t+\int_{0}^{x} q_{0} f(x) d x \geqq O\left(e^{\alpha x}\right) \quad(x \rightarrow+\infty),
$$

from which it follows that

$$
\int_{0}^{x} Q^{*}(D) f^{\prime}(t) d t \geqq O\left(e^{\alpha x}\right) \quad(x \rightarrow+\infty) .
$$

Applying our theorem to $f^{\prime}(x)$ with $k=1$ we deduce that $f^{\prime \prime}(x)=o\left(e^{\alpha x}\right)$ as $(x \rightarrow+\infty)$. We may show successively that $f^{(k)}(x)=o\left(e^{\alpha x}\right)$ as $(x \rightarrow+\infty)$ for $k=3, \cdots, n-2$.

Let us now prove our theorem for $k=1$. We set

$$
F(x)=\frac{1}{(n-1) !} \int_{0}^{x}(x-t)^{n-1} Q(D) f(t) d t .
$$

Integrating by parts we may show that

$$
F(x)=q_{n} f(x)+\sum_{j=0}^{n-1} \frac{q_{j}}{(n-j-1) !} \int_{0}^{x}(x-t)^{n-j-1} f(t) d t+\Omega(x),
$$

where $\Omega(x)$ is a polynomial of degree $n-1$. By assumption 2 and Lemma $28 \mathrm{~b}$ we have

$$
F(x)=o\left(e^{\alpha x}\right) \quad(x \rightarrow+\infty)
$$

On the other hand

$$
F^{\prime \prime}(x)=\frac{1}{(n-4) !} \int_{0}^{t}(x-t)^{n-4} d t \int_{0}^{t} Q(D) f(u) d u .
$$

Hence by assumption 3

$$
F^{\prime \prime}(x) \geqq O\left(e^{\alpha x}\right) \quad(x \rightarrow+\infty) .
$$

Applying Theorem 31 a to $F(x)$ we see that

$$
F^{\prime}(x)=o\left(e^{\alpha x}\right) \quad(x \rightarrow+\infty) .
$$

But

$$
F^{\prime}(x)=q_{n} f^{\prime}(x)+o\left(e^{\alpha x}\right) \quad(x \rightarrow+\infty),
$$

and so, since $q_{n} \neq 0$, we have 


$$
f^{\prime}(x)=o\left(e^{\alpha x}\right)
$$

$(x \rightarrow+\infty)$,

as desired.

THEOREM 31c. Let $Q(D)$ be a linear differential operator of degree $n$ with constant coefficients

$$
Q(D)=q_{n} D^{n}+q_{n-1} D^{n-1}+\cdots+q_{0} \quad\left(q_{n} \neq 0\right) .
$$

If

1.

2.

$$
f(x) \in C^{n}(-\infty<x \leqq 0)
$$

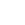

3.

$$
\begin{array}{ll}
f(x)=o\left(e^{\alpha x}\right), \quad \alpha<0, & (x \rightarrow-\infty), \\
\int_{x}^{0} Q(D) f(t) d t \geqq O\left(e^{\alpha x}\right) & (x \rightarrow-\infty)
\end{array}
$$

then

$$
f^{(k)}(x)=o\left(e^{\alpha x}\right) \quad(x \rightarrow-\infty ; k=1,2, \cdots, n-2) .
$$

32. Determining function in $L_{p}, p>1$. Let $L_{p}$ denote, as is usual, the class of functions $\phi(t)$ such that

$$
\int_{-\infty}^{\infty}|\phi(t)|^{p} d t<\infty
$$

Further let $q$ be the conjugate index of $p$ defined by the equation

$$
\frac{1}{q}+\frac{1}{p}=1
$$

We shall require the following well known result, see $[17$, p. 33].

THEOREM I. Let the functions $\phi_{n}(t) \in L_{p}, n=1,2, \cdots$. If there exists a constant $M$ such that

$$
\int_{-\infty}^{\infty}\left|\phi_{n}(t)\right|^{p} d t<M \quad(n=1,2, \cdots)
$$

then there exists a subsequence of the $n^{\prime} s, n_{1}, n_{2}, n_{3}, \cdots$, and a function $\phi(t) \in L_{p}$ such that for every function $\psi(t) \in L_{q}$ we have

$$
\lim _{i \rightarrow \infty} \int_{-\infty}^{\infty} \psi(t) \phi_{n_{i}}(t) d t=\int_{-\infty}^{\infty} \psi(t) \phi(t) d t .
$$

Using this we may prove the following representation theorem.

Theorem 32. Let $\alpha_{1}<c<\alpha_{2}$ if $G(t) \in$ class I and let $c<\alpha_{2}$ if $G(t) \in$ class II or III. Necessary and sufficient conditions that 


$$
f(x)=\int_{-\infty}^{\infty} G(x-t) e^{c t} \phi(t) d t,
$$

where $\phi(t) \in L_{p}, p>1$, are
A.
$f(x) \in C^{\infty}(-\infty<x<\infty)$,
B.

$$
\int_{-\infty}^{\infty}\left|e^{-c x} P_{n}(D) f(x)\right|^{p} d x \leqq M \quad(n=0,1,2, \cdots),
$$

where $M$ is a constant independent of $n$.

The necessity of condition $A$ is obvious. The equation

$$
e^{-c x} P_{n}^{\dagger}(D) f(x)=\int_{-\infty}^{\infty} G_{n}^{\dagger}(x-t) e^{-c(x-t)} \phi(t) d t,
$$

together with Hölder's inequality, implies that

$$
\begin{aligned}
\left|e^{-c x} P_{n}^{\#}(D) f(x)\right|^{p} & \\
& \leqq\left[\int_{-\infty}^{\infty} e^{-c(x-t)} G_{n}^{\#}(x-t)|\phi(t)|^{p} d t\right]\left[\int_{-\infty}^{\infty} e^{-c(x-t)} G_{n}(x-t) d t\right]^{p / q} .
\end{aligned}
$$

Since $\alpha_{1}<c<\alpha_{2}$ if $G(t) \in$ class I, and $c<\alpha_{2}$ if $G^{*}(t) \in$ class II or III, we have in both cases

$$
\int_{-\infty}^{\infty} e^{-c(x-t)} G_{n}^{t}(x-t) d t=\left[\prod_{n+1}^{\infty}\left(1-\frac{c}{a_{k}}\right) e^{c / a_{k}}\right]^{-1} .
$$

Therefore there exists a constant $A$ such that

$$
\int_{-\infty}^{\infty} e^{-c(x-t)} G_{n}^{t}(x-t) d t \leqq A \quad(n=0,1,2, \cdots) .
$$

Thus

$$
\int_{-\infty}^{\infty}\left|e^{-c x} P_{n}^{*}(D) f(x)\right| p d x \leqq A^{p / q} \int_{-\infty}^{\infty} d x \int_{-\infty}^{\infty} e^{-c(x-t)} G_{n}^{t}(x-t)|\phi(t)|^{p} d t .
$$

Since the integrand of this iterated integral is non-negative we may invert the order of the integrations to obtain

$$
\begin{aligned}
\int_{-\infty}^{\infty}\left|e^{-c x} P_{n}^{\#}(D) f(x)\right|^{p} d x & \leqq A^{p / q} \int_{-\infty}^{\infty}|\phi(t)|^{p} d t \int_{-\infty}^{\infty} e^{-c(x-t)} G_{n}^{*}(x-t) d x \\
& \leqq A^{p} \int_{-\infty}^{\infty}|\phi(t)|^{p} d t
\end{aligned}
$$


We have thus demonstrated the necessity of condition $\mathrm{B}$.

We turn now to the sufficiency of our conditions. By Hölder's inequality

$$
\left|\int_{0}^{x} P_{n}^{\#}(D) f(x) d x\right| \leqq\left|\int_{0}^{x}\right| e^{-c x} P_{n}^{\#}(D) f(x)|p d x|^{1 / p}\left|\int_{0}^{x} e^{c x q} d x\right|^{1 / q},
$$

from which it follows that

$$
\int_{0}^{x} P_{n}^{\sharp}(D) f(x) d x=O\left(e^{c x}+1\right) \quad(x \rightarrow \pm \infty ; n=0,1, \cdots) .
$$

Let $F(x)=\int_{0}^{x} f(t) d t$. Setting $n=0$ in equation (1) and recalling the inequalities satisfied by $c$, we obtain

$$
\begin{aligned}
F(x) & =o\left(e^{\alpha_{2} x}\right) & & (x \rightarrow+\infty) \\
& =o\left(e^{\alpha_{1} x}\right) & & (x \rightarrow-\infty)
\end{aligned}
$$

if $G(t) \in$ class I, and

$$
\begin{aligned}
F(x) & =o\left(e^{\alpha_{2} x}\right) & & (x \rightarrow+\infty) \\
& =o\left(e^{\theta x}\right) & & (x \rightarrow-\infty)
\end{aligned}
$$

where $\theta<\min (c, 0)$ if $G(t) \in$ class II or class III. For general $n$ equation (1) implies that

$$
\begin{aligned}
\int_{0}^{x} P_{n}^{t}(D) D F(t) d t & =O\left(e^{\alpha_{2} x}\right) & & (x \rightarrow+\infty) \\
& =O\left(e^{\alpha_{1} x}\right) & & (x \rightarrow-\infty)
\end{aligned}
$$

if $G(t)$ class I, and

$$
\begin{aligned}
\int_{0}^{x} P_{n}^{\#}(D) D F(t) d t & =O\left(e^{\alpha_{2} x}\right) & & (x \rightarrow+\infty) \\
& =O\left(e^{\theta x}\right) & & (x \rightarrow-\infty)
\end{aligned}
$$

if $G(t) \in$ class II or class III. Applying Theorems $31 \mathrm{~b}$ and $31 \mathrm{c}$ to $F(x)$ we obtain

$$
\begin{aligned}
f^{(n)}(x) & =o\left(e^{\alpha_{2} x}\right) & & (x \rightarrow+\infty ; n=0,1, \cdots) \\
& =o\left(e^{\alpha_{1} x}\right) & & (x \rightarrow-\infty ; n=0,1, \cdots)
\end{aligned}
$$

if $G(t) \in$ class I, and

$$
\begin{aligned}
f^{(n)}(x) & =o\left(e^{\alpha_{2} x}\right) & & (x \rightarrow+\infty ; n=0,1, \cdots) \\
& =o\left(e^{\theta x}\right) & & (x \rightarrow-\infty ; n=0,1, \cdots)
\end{aligned}
$$

if $G(t) \in$ class II or class III. 
By Theorem 29c with $m=\nu_{1}$

$$
P_{\nu_{1}}^{\#}(D) f(x)=\lim _{n \rightarrow \infty} \int_{-\infty}^{\infty} G_{\nu_{1}}^{\#}(x-t) P_{n}^{\#}(D) f(t) d t,
$$

or

$$
P_{\nu_{1}}^{\#}(D) f(x)=\lim _{n \rightarrow \infty} \int_{-\infty}^{\infty} G_{\nu_{1}}^{\#}(x-t) e^{c t}\left[e^{-c t} P_{n}^{\#}(D) f(t)\right] d t
$$

By condition B and Theorem I there exists a set of indices $n_{1}, n_{2}, n_{3}, \cdots$ and a function $\phi(t) \in L_{p}$ such that for every $\psi(t) \in L_{q}$

$$
\lim _{t \rightarrow \infty} \int_{-\infty}^{\infty} \psi(t)\left[e^{-c t} P_{n_{\mathfrak{\imath}}}^{\#}(D) f(t)\right] d t=\int_{-\infty}^{\infty} \psi(t) \phi(t) d t .
$$

By Theorem $9 \mathrm{~b} G_{\nu_{1}}^{\#}(x-t) e^{c t}$ is for each fixed value of $x, \in L_{p}$, therefore,

$$
\begin{gathered}
P_{\nu_{1}}^{\#}(D) f(x)=\lim _{t \rightarrow \infty} \int_{-\infty}^{\infty} G_{\nu_{1}}^{\#}(x-t) e^{c t}\left[e^{-c t} P_{n_{i}}^{\#}(D) f(t)\right] d t, \\
P_{\nu_{1}}^{\#}(D) f(x)=\int_{-\infty}^{\infty} G_{\nu_{1}}^{\#}(x-t) e^{c t} \phi(t) d t .
\end{gathered}
$$

By Lemma 30 we have

$$
f(x)=g_{1} * g_{2} * \cdots * g_{\nu_{1}} * \int_{-\infty}^{\infty} G_{\nu_{1}}^{\#}(x-t) e^{c t} \phi(t) d t .
$$

Since $\phi \in L_{p}$ this iterated integral is absolutely convergent and may be inverted to give

$$
f(x)=\int_{-\infty}^{\infty} G^{\sharp}(x-t) e^{c t} \phi(t) d t
$$

as desired.

33. Determining function bounded. Let $L_{\infty}$ denote, as is customary, the class of functions $\phi(t)$ essentially bounded for $(-\infty<t<\infty)$. We need the following result. See $[17$, p. 33].

THEOREM II. Let the functions $\phi_{n}(t) \in L_{\infty}, n=1,2, \cdots$. If there exists a constant $M$ such that

$$
\underset{-\infty<t<\infty}{\text { essential sup }}\left|\phi_{n}(t)\right| \leqq M \quad(n=1,2, \cdots)
$$

then there exists a subsequence of the $n$ 's, $n_{1}, n_{2}, \cdots$, and a function $\phi(t) \in L_{\infty}$ such that for every function $\psi(t) \in L$ we have 


$$
\lim _{i \rightarrow \infty} \int_{-\infty}^{\infty} \psi(t) \phi_{n_{i}}(t) d t \int_{-\infty}^{\infty} \psi(t) \phi(t) d t
$$

Using this we may prove the following theorem.

Theorem 33. Let $\alpha_{1}<c<\alpha_{2}$ if $G(t) \in$ class I, and let $c<\alpha_{2}$ if $G(t) \in$ class II or III. Necessary and sufficient conditions that

$$
f(x)=\int_{-\infty}^{\infty} G(x-t) e^{c t} \phi(t) d t
$$

where $\phi(t) \in L_{\infty}$, are

A.

$$
\begin{aligned}
& f(x) \in C^{\infty}(-\infty<x<\infty), \\
& \sup _{-\infty<x<\infty}\left|e^{-c x} P_{n}(D) f(x)\right| \leqq M \quad(n=0,1,2, \cdots),
\end{aligned}
$$

B.

where $M$ is a constant independent of $n$.

The necessity of condition A is obvious. For condition B assume that $|\phi(t)| \leqq N$ almost everywhere. From the equation

$$
e^{-c x} P_{n}^{\sharp}(D) f(x)=\int_{-\infty}^{\infty} G_{n}^{\#}(x-t) e^{-c(x-t)} \phi(t) d t,
$$

we may then deduce

$$
\begin{aligned}
\left|e^{-c x} P_{n}^{\#}(D) f(x)\right| & \leqq N \int_{-\infty}^{\infty} G_{n}^{\#}(x-t) e^{-c(x-t)} d t \\
& \leqq N\left[\prod_{n+1}^{\infty}\left(1-\frac{c}{a_{k}}\right) e^{c / a_{k}}\right]^{-1}<M
\end{aligned}
$$

for some number $M$. This completes the proof of the necessity.

To establish the sufficiency of our conditions, we proceed just as in $\$ 32$. By condition $\mathrm{B}$

$$
\left|P_{n}^{\sharp}(D) f(x)\right| \leqq M e^{c x} \quad(n=0,1,2, \cdots) .
$$

Using Theorem $31 \mathrm{~b}$ and $31 \mathrm{c}$, we see that if $G(t) \in$ class I then

$$
\begin{aligned}
f^{(n)}(x) & =o\left(e^{\alpha_{2} x}\right) & & (x \rightarrow+\infty) \\
& =o\left(e^{\alpha_{1} x}\right) & & (x \rightarrow-\infty),
\end{aligned}
$$

and if $G(t) \in$ class II or III then

$$
\begin{aligned}
f^{(n)}(x) & =o\left(e^{\alpha_{2} x}\right) & & (x \rightarrow+\infty) \\
& =o\left(e^{\theta x}\right) & & (x \rightarrow-\infty),
\end{aligned}
$$


where $\theta<\min (c, 0)$. By Theorem 29c

$$
P_{\nu_{1}}^{\#}(D) f(x)=\lim _{n \rightarrow \infty} \int_{-\infty}^{\infty} G_{\nu_{1}}^{\#}(x-t) P_{n}^{\#}(D) f(t) d t,
$$

which we may rewrite as

$$
P_{\nu_{1}}^{\#}(D) f(x)=\lim _{n \rightarrow \infty} \int_{-\infty}^{\infty} G_{\nu_{1}}^{\#}(x-t) e^{c t}\left[e^{-c t} P_{n}^{\#}(D) f(t)\right] d t .
$$

By condition B and Theorem II there exists a set of indices $n_{1}, n_{2}, \cdots$ and a function $\phi(t) \in L_{\infty}$ such that for every $\psi(t) \in L$

$$
\lim _{i \rightarrow \infty} \int_{-\infty}^{\infty} \psi(t)\left[e^{-c t} P_{n_{i}}^{\#}(D) f(t) d t\right]=\int_{-\infty}^{\infty} \psi(t) \phi(t) d t
$$

For each fixed value of $x, G_{\nu_{1}}^{\#}(x-t) e^{c t} \in L$; therefore

$$
\begin{aligned}
P_{\nu_{1}}^{*}(D) f(x) & =\lim _{t \rightarrow \infty} \int_{-\infty}^{\infty} G_{\nu_{1}}^{\#}(x-t) e^{c t}\left[e^{-c t} P_{n_{i}}^{\#}(D) f(t)\right] d t \\
& =\int_{-\infty}^{\infty} G_{\nu_{1}}^{\#}(x-t) e^{c t} \phi(t) d t, \quad(-\infty<x<\infty)
\end{aligned}
$$

By Lemma 30 we have

$$
f(x)=g_{1} * g_{2} * \cdots * g_{v_{1}} * \int_{-\infty}^{\infty} G_{\nu_{1}}^{*}(x-t) e^{c t} \phi(t) d t .
$$

Since $\phi(t) \in L_{\infty}$ this iterated integral is absolutely convergent and the order of the integrations may be inverted to yield as desired,

$$
f(x)=\int_{-\infty}^{\infty} G^{\sharp}(x-t) e^{c t} \phi(t) d t
$$

34. Determining function of bounded total variation. We require the following result, see $[17$, p. 26$]$.

THEOREM III. If $\alpha_{1}(t), \alpha_{2}(t), \cdots$ are a set of functions uniformly bounded and of uniformly bounded variation in every finite interval, then there exists a set of indices $n_{1}, n_{2}, \cdots$ and a function $\alpha(t)$ of bounded variation in every finite interval such that

$$
\lim _{i \rightarrow \infty} \alpha_{n_{i}}(t)=\alpha(t) \quad(-\infty<t<\infty) .
$$

If $\phi(t)$ is any continuous function then

$$
\lim _{i \rightarrow \infty} \int_{a}^{b} \phi(t) d \alpha_{n_{i}}(t)=\int_{a}^{b} \phi(t) d \alpha(t) .
$$


If the $\alpha_{k}(t)$ are of uniformly bounded total variation in $(-\infty<t<\infty)$, then $\alpha(t)$ is of bounded total variation there. In this case if $\phi(t)$ is a continuous function such that $\phi( \pm \infty)=0$ then

$$
\lim _{i \rightarrow \infty} \int_{-\infty}^{\infty} \phi(t) d \alpha_{n_{i}}(t)=\int_{-\infty}^{\infty} \phi(t) d \alpha(t) .
$$

Theorem 34. Let $\alpha_{1}<c<\alpha_{2}$ if $G(t) \in$ class I and let $c<\alpha_{2}$ if $G(t) \in$ class II or III. Necessary and sufficient conditions that $f(x)=\int_{-\infty}^{\infty} G(x-t) e^{c t} d \alpha(t)$ where $\alpha(t)$ is of bounded total variation in $(-\infty<t<\infty)$ are

A.

$$
\begin{aligned}
& f(x) \in C^{\infty}(-\infty<x<\infty), \\
& \int_{-\infty}^{\infty} e^{-c x}\left|P_{n}(D) f(x)\right| d x \leqq M \quad(n=0,1, \cdots) .
\end{aligned}
$$

B.

The necessity of condition $\mathrm{A}$ is obvious. From the equation

$$
e^{-c x} P_{n}^{\sharp}(D) f(x)=\int_{-\infty}^{\infty} G_{n}^{\#}(x-t) e^{-c(x-t)} d \alpha(t)
$$

we may deduce the inequality

$$
\left|e^{-c x} P_{n}^{\sharp}(D) f(x)\right| \leqq \int_{-\infty}^{\infty} G_{n}^{\#}(x-t) e^{-c(x-t)} d V(t)
$$

where $V(t)=\operatorname{Var}_{-\infty<u \leqq t}[\alpha(u)]$. It follows that

$$
\int_{-\infty}^{\infty}\left|e^{-c x} P_{n}^{*}(D) f(x)\right| d x \leqq \int_{-\infty}^{\infty} d x \int_{-\infty}^{\infty} G_{n}^{\#}(x-t) e^{-c(x-t)} d V(t) .
$$

Since the integrand is non-negative the order of the integrations may be inverted to give

$$
\begin{aligned}
\int_{-\infty}^{\infty}\left|e^{-c x} P_{n}^{\#}(D) f(x)\right| d x & \leqq V(\infty) \int_{-\infty}^{\infty} G_{n}^{\#}(x-t) e^{-c(x-t)} d x \\
& \leqq V(\infty)\left[\prod_{n+1}^{\infty}\left(1-\frac{c}{a_{k}}\right) e^{c / a_{k}}\right]^{-1}
\end{aligned}
$$

and from this inequality the necessity of condition $\mathrm{B}$ is evident.

To establish the sufficiency of our conditions we proceed as before. We have

$$
\begin{aligned}
\left|\int_{0}^{x} P_{n}^{\#}(D) f(t) d t\right| & \leqq\left[\max _{0 \leqq t \leqq x} e^{c t}\right]\left|\int_{0}^{x} e^{-c t} P_{n}^{\sharp}(D) f(t) d t\right| \\
& \leqq\left[\max _{0 \leqq t \leqq x} e^{c t}\right] \int_{-\infty}^{-\infty}\left|e^{-c t} P_{n}^{t}(D) f(t)\right| d t \leqq M\left[e^{c x}+1\right]
\end{aligned}
$$


If, as in the proof of Theorem 32, we apply Theorem $31 \mathrm{~b}$ to the function $F(x)=\int_{0}^{x} f(t) d t$ we find that if $G(t) \in$ class I then

$$
\begin{aligned}
f^{(n)}(x) & =o\left(e^{\alpha_{2} x}\right) & & (x \rightarrow+\infty ; n=0,1,2, \cdots) \\
& =o\left(e^{\alpha_{1} x}\right) & & (x \rightarrow-\infty ; n=0,1,2, \cdots),
\end{aligned}
$$

and if $G(t)$ belongs to class II or class II, then

$$
\begin{aligned}
f^{(n)}(x) & =o\left(e^{\alpha_{2} x}\right) & & (x \rightarrow+\infty ; n=0,1,2, \cdots) \\
& =o\left(e^{\theta x}\right) & & (x \rightarrow-\infty ; n=0,1,2, \cdots),
\end{aligned}
$$

where $\theta<\min (0, c)$. By Theorem 29c with $m=\nu_{1}$ we have

$$
P_{\nu_{1}}^{\#}(D) f(x)=\lim _{n \rightarrow \infty} \int_{-\infty}^{\infty} G_{\nu_{1}}^{\#}(x-t) e^{c t}\left[e^{-c t} P_{n}^{\#}(D) f(t)\right] d t .
$$

By condition B and Theorem III there exists a set of indices $n_{1}, n_{2}, \cdots$ and a function $\alpha(t)$ of bounded total variation such that

$$
\lim _{i \rightarrow \infty} \int_{0}^{x} e^{-c t} P_{n_{i}}^{t}(D) f(t) d t=\alpha(x) \quad(-\infty<x<\infty),
$$

and such that if $\psi(t)$ is a continuous function and $\psi( \pm \infty)=0$, then

$$
\lim _{i \rightarrow \infty} \int_{-\infty}^{\infty} \psi(t)\left[e^{-c t} P_{n_{i}}^{\sharp}(D) f(t)\right] d t=\int_{-\infty}^{\infty} \psi(t) d \alpha(t)
$$

For each value of $x, G(x-t) e^{c t}$ is a continuous function vanishing at $\pm \infty$; hence

$$
\begin{aligned}
P_{\nu_{1}}^{\#}(D) f(x) & =\lim _{i \rightarrow \infty} \int_{-\infty}^{\infty} G_{\nu_{1}}^{\#}(x-t) e^{c t}\left[e^{-c t} P_{n_{i}}^{\#}(D) f(t)\right] d t \\
& =\int_{-\infty}^{\infty} G_{\nu_{1}}^{\#}(x-t) e^{c t} d \alpha(t) .
\end{aligned}
$$

By Lemma 30 we have

$$
f(x)=g_{1} * g_{2} * \cdots * g_{\nu_{1}} * \int_{-\infty}^{\infty} G_{\nu_{1}}^{\#}(x-t) e^{c t} d \alpha(t) .
$$

Since $\alpha(t)$ is of bounded total variation this iterated integral converges absolutely and may be inverted to give

$$
f(x)=\int_{-\infty}^{\infty} G^{\sharp}(x-t) e^{c t} d \alpha(t),
$$

as desired.

35. The positiveness of certain differential operators. In this section we 
shall derive certain results which we shall need later.

THEOREM 35a. If.

1.

$$
\begin{aligned}
& G(t) \in \text { class } \mathrm{I}, \\
& \text { 2. } f(x) \in C^{\infty} \\
& (-\infty<x<\infty) \text {, } \\
& (x \rightarrow+\infty) \\
& =o\left(e^{\alpha_{1} x}\right) \\
& (x \rightarrow-\infty) \text {, }
\end{aligned}
$$$$
\text { 3. } \quad f(x)=o\left(e^{\alpha_{2} x}\right)
$$

4.

$$
P_{n_{i}}(D) f(x) \geqq 0 \quad(-\infty<x<\infty ; i=1,2, \cdots),
$$

where $n_{1}<n_{2}<\cdots$ is any increasing sequence of integers, then

$$
e^{b^{\prime} D} \prod_{i=1}^{n}\left(1-\frac{D}{a_{k_{i}}}\right) e^{D / a_{k_{i}} f(x) \geqq 0} \quad(-\infty<x<\infty)
$$

where $b^{\prime}$ is any real constant and the $\left\{a_{k_{i}}\right\}_{i=1}^{n}$ are any selection from the $\left\{a_{k}\right\}_{k=1}^{\infty}$.

It follows from assumption 4 that

$$
\begin{array}{ll}
\int_{0}^{x} P_{n_{i}}^{\sharp}(D) f(t) d t \geqq 0 & (x \geqq 0), \\
\int_{x}^{0} P_{n_{i}}(D) f(t) d t \geqq 0 & (x \leqq 0) .
\end{array}
$$

This together with assumption 3 and Theorems $31 \mathrm{~b}$ and $31 \mathrm{c}$ implies that

$$
\begin{aligned}
f^{(n)}(x) & =o\left(e^{\alpha_{2} x}\right) & & (x \rightarrow+\infty ; n=0,1, \cdots) \\
& =o\left(e^{\alpha_{1} x}\right) & & (x \rightarrow-\infty ; n=0,1, \cdots) .
\end{aligned}
$$

Choose $n_{j}$ so large that

$$
\prod_{i=1}^{n}\left(1-\frac{s}{a_{k_{i}}}\right) e^{s / a_{k_{i}}}=P_{n_{j}}^{\#}(s) / \prod_{i=1}^{m}\left(1-\frac{s}{a_{l_{i}}}\right) e^{s / a_{l_{i}}}
$$

where the $\left\{a_{l_{i}}\right\}_{i=1}^{m}$ are certain additional elements from the $\left\{a_{k}\right\}_{k=1}^{\infty}$. Using equations (1) and Lemma 30 we have

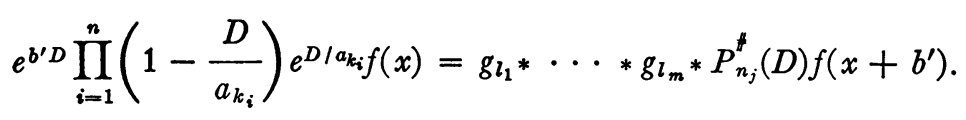

Since the functions $g_{i}$ are non-negative and since by assumption 4 we have $P_{n_{j}}^{*}(D) f\left(x+b^{\prime}\right) \geqq 0(-\infty<x<\infty)$, it follows that

$$
e^{b^{\prime} D} \prod_{i=1}^{n}\left(1-\frac{D}{a_{k_{i}}}\right) e^{D / a_{k_{i}} f(x) \geqq 0} \quad(-\infty<x<\infty),
$$

which is what we wished to prove. 
THEOREM 35b. If

1.

2.

3.

4 .
$G(t) \in$ class II or III,

$$
f(x) \in C^{\infty}
$$$$
\left(\gamma_{c}<x<\infty\right)
$$$$
f(x)=o\left(e^{\alpha_{2} x}\right)
$$$$
(x \rightarrow+\infty) \text {, }
$$$$
\prod_{i=1}^{n_{i}}\left(1-\frac{D}{a_{k_{i}}}\right) f(x) \geqq 0
$$

where $n_{1}<n_{2}<\cdots$ is any increasing sequence of integers, then

$$
\prod_{i=1}^{n}\left(1-\frac{D}{a_{k_{i}}}\right) f(x) \geqq 0 \quad\left(\gamma_{c}<x<\infty\right)
$$

where the $\left\{a_{k_{i}}\right\}_{i=1}^{n}$ are a selection from the $\left\{a_{k}\right\}_{k=1}^{\infty}$.

This is proved exactly as Theorem 35 a.

36. Determining function nondecreasing (class I). Unlike the representation theorems which we have so far proved the theorems of this and the succeeding sections require very different proofs for each type of kernel.

THEOREM 36. Let $G(t) \in$ class I. Necessary and sufficient conditions that a function $f(x)$ defined for $(-\infty<x<\infty)$ be representable in the form

$$
f(x)=\int_{-\infty}^{\infty} G(x-t) d \beta(t)
$$

where $\beta(t) \in \uparrow$ are
A.
$f(x) \in C^{\infty}$
$(-\infty<x<\infty)$,
B. $f(x)=o\left(e^{\alpha_{2} x}\right)$
$(x \rightarrow+\infty)$
$=o\left(e^{\alpha_{1} x}\right)$
$(x \rightarrow-\infty)$,
C. $\quad P_{n}(D) f(x) \geqq 0$
$(-\infty<x<\infty ; n=0,1,2, \cdots)$.

The necessity of condition $\mathrm{A}$ is evident and that of condition $\mathrm{B}$ follows from Theorem 28a. The equation

$$
P_{n}(D) f(x)=\int_{-\infty}^{\infty} G_{n}(x-t) d \beta(t)
$$

and the fact that $\beta(t) \in \uparrow$ implies that

$$
P_{n}(D) f(x) \geqq 0 \quad(-\infty<x<\infty ; n=0,1, \cdots),
$$

so that condition $\mathrm{C}$ is also necessary.

In order to prove the sufficiency of our conditions we note that in view of Theorem 35 a we may assume that 


$$
\begin{aligned}
& P_{\nu_{1}}^{\#}(D)=\left[\left(1-\frac{D}{\alpha_{1}}\right) e^{D / \alpha_{1}}\right]^{\mu_{1}}\left[\left(1-\frac{D}{\alpha_{2}}\right) e^{D / \alpha_{2}}\right]^{\mu_{2}}, \\
& P_{\nu_{2}}^{*}(D)=\left[\left(1-\frac{D}{\alpha_{1}}\right) e^{D / \alpha_{1}}\right]^{\mu_{1}+1}\left[\left(1-\frac{D}{\alpha_{2}}\right) e^{D / \alpha_{2}}\right]^{\mu_{2}+1} .
\end{aligned}
$$

We define

$$
\beta_{n}(u)=\int_{0}^{u} P_{n}^{*}(D) f(t) d t \quad(n=0,1,2, \cdots)
$$

Clearly $\beta_{n}(u) \in \uparrow$ for every $n$. If we set

$$
\frac{1}{A}=\min _{-\infty<u<\infty}\left[\min _{t} \frac{G_{\nu_{1}}^{\#}(t)}{G_{\nu_{1}}^{\#}(u)}, t \text { between } 0 \text { and } u\right],
$$

it is easily verified that from the shape of the curve $G_{\nu_{1}}^{*}(t)$, see $\$ 10$, that

$$
\frac{1}{A}=G_{\nu_{1}}^{\#}(0)\left[\max _{-\infty<t<\infty} G_{\nu_{1}}^{\#}(t)\right]^{-1} \text {, }
$$

and hence $1 \leqq A<\infty$.

By elementary estimations

$$
\begin{aligned}
\int_{-\infty}^{\infty} G_{\nu_{1}}^{\#}(-t) P_{k}^{\#}(D) f(t) d t & \geqq\left[\min _{t \in[0, u]} G_{\nu_{1}}^{\#}(-t)\right]\left|\int_{0}^{u} P_{k}^{\#}(D) f(t) d t\right| \\
& \geqq \frac{1}{A} G_{\nu_{1}}^{\#}(-u)\left|\int_{0}^{u} P_{k}^{\#}(D) f(t) d t\right|,
\end{aligned}
$$

which implies that

$$
\left|\beta_{k}(u)\right| \leqq A\left[G_{\nu_{1}}^{\#}(-u)\right]^{-1} \int_{-\infty}^{\infty} G_{\nu_{1}}^{\#}(-t) P_{k}^{\sharp}(D) f(t) d t .
$$

By Theorem $29 \mathrm{~b}$ if $k \geqq \nu_{2}$ we have

$$
\int_{-\infty}^{\infty} G_{\nu_{1}}^{\#}(-t) P_{k}^{\#}(D) f(t) d t=\int_{-\infty}^{\infty} G_{k}^{\#}(-t) P_{\nu_{1}}^{\#}(D) f(t) d t .
$$

Conditions $\mathrm{B}$ and $\mathrm{C}$ together with Theorems $31 \mathrm{~b}$ and $31 \mathrm{c}$ imply that there exists a constant $E$ such that $0 \leqq P_{\nu_{1}}^{\#}(D) f(t) \leqq E\left[e^{\alpha_{2} t}+e^{\alpha_{1} t}\right](-\infty<t<\infty)$. Hence

$$
\int_{-\infty}^{\infty} G_{k}^{\sharp}(-t) P_{\nu_{1}}^{\#}(D) f(t) d t \leqq \frac{E}{\prod_{k+1}^{\infty}\left(1-\frac{\alpha_{2}}{a_{j}}\right) e^{\alpha_{2} / a_{j}}}+\frac{E}{\prod_{k+1}^{\infty}\left(1-\frac{\alpha_{1}}{a_{j}}\right) e^{\alpha_{1} / a_{j}}},
$$


and consequently there exists a constant $F$ such that

$$
\left|\int_{-\infty}^{\infty} G_{k}^{t}(-t) P_{\nu_{1}}^{\sharp}(D) f(t) d t\right| \leqq F \quad\left(k=\nu_{2}, \nu_{2}+1, \cdots\right) .
$$

It follows that

$$
\left|\beta_{k}(u)\right| \leqq A F\left[G_{\nu_{1}}^{*}(-u)\right]^{-1} \quad\left(k=\nu_{2}, \nu_{2}+1, \cdots\right) .
$$

Since $\beta_{k}(t) \in \uparrow$ for every $k$, inequality (3) implies that the functions $\beta_{k}(u)$ are uniformly bounded and of uniformly bounded variation in every finite interval. We may therefore, by Theorem III, select a subsequence $\left\{\beta_{k_{1}}(u)\right\}$ which converges to a function $\beta(u)$ of bounded variation in every finite interval. Evidently $\beta(u) \in \uparrow$ and $|\beta(u)| \leqq A F\left[G_{\nu_{1}}^{\#}(-u)\right]^{-1}$. By Theorem 29c with $m=\nu_{2}$ we have

$$
\begin{aligned}
P_{\nu_{2}}^{\#}(D) f(x) & =\lim _{k \rightarrow \infty} \int_{-\infty}^{\infty} G_{\nu_{2}}^{\#}(x-t) P_{k}^{\#}(D) f(t) d t \\
& =\lim _{i \rightarrow \infty} \int_{-\infty}^{\infty} G_{\nu_{2}}^{\#}(x-t) d \beta_{k_{i}}(t) .
\end{aligned}
$$

By Theorem 9b we have, recalling equations (1) and (2),

$$
\begin{aligned}
{\left[G_{\nu_{1}}^{\#}(-t)\right]^{-1} } & =O\left(e^{\alpha_{2} t}\right) & & (t \rightarrow+\infty) \\
& =O\left(e^{\alpha_{1} t}\right) & & (t \rightarrow-\infty), \\
G_{\nu_{2}}^{\#}(x-t), \frac{d}{d t} G_{\nu_{2}}^{\#}(x-t) & =O\left(e^{-\left(\alpha_{2}+\epsilon\right) t}\right) & & (t \rightarrow+\infty) \\
& =O\left(e^{-\left(\alpha_{1}-\epsilon\right) t}\right) & & (t \rightarrow-\infty),
\end{aligned}
$$

where $\epsilon$ is some positive number and $x$ is fixed. These equations together with inequality (3) enable us to integrate equation (4) by parts to obtain

$$
P_{\nu_{2}}^{\#}(D) f(x)=\lim _{i \rightarrow \infty}-\int_{-\infty}^{\infty} \beta_{k_{i}}(t)\left[\frac{d}{d t} G_{\nu_{2}}^{\#}(x-t)\right] d t .
$$

These same results enable us to apply Lebesgue's general limit theorem to conclude that

$$
P_{\nu_{2}}^{\#}(D) f(x)=-\int_{-\infty}^{\infty} \beta(t) \frac{d}{d t}\left[G_{\nu_{2}}^{\#}(x-t)\right] d t .
$$

Integrating by parts again we have

$$
P_{\nu_{2}}^{\#}(D) f(x)=\int_{-\infty}^{\infty} G_{\nu_{2}}^{\#}(x-t) d \beta(t) .
$$


By conclusion A of Lemma 30

$$
f(x)=g_{1} * g_{2} * \cdots * g_{\nu_{2}} * \int_{-\infty}^{\infty} G_{\nu_{2}}^{*}(x-t) d \beta(t) .
$$

Since $\beta(t) \in \uparrow$ and since the functions $g_{i}$ and $G_{\nu_{2}}$ are non-negative, this iterated integral is absolutely convergent, and the order of integrations may be inverted to give

$$
f(x)=\int_{-\infty}^{\infty} G^{*}(x-t) d \beta(t) .
$$

37. Determining function nondecreasing (class II). Let $G^{\sharp}(t) \in$ class II. We define, see $\$ 20$,

$$
\chi_{m, n}^{\#}(t)=-\log G_{m, n}^{t}(t) \quad(-\infty<t<\infty) .
$$

By convention $(d / d t)^{i} \chi_{m, n}^{*}(t)(i=0,1,2)$ is to be $+\infty$ whenever $G_{m, n}^{\#}(t)=0$.

THEOREM IV. If $\chi_{m, n}^{\#}(t)$ is defined as in equation (1) then, for $n-m \geqq 4$,

$$
\left(\frac{d}{d t}\right)^{2} \chi_{m, n}^{*}(t) \geqq 0 \quad(-\infty<t<\infty) .
$$

This result is stated by Schoenberg [13]; see also Pólya and Szegö [12, vol. II, pp. 52-53]. Using this we may prove the following theorems.

THEOREM $37 \mathrm{a}$. If

1. G $G^{*} \in$ class II,

2. $\chi_{m, n}^{\#}(t)$ is defined as in (1),

3. $L(t)$ is defined as in $\$ 20$,

then given $\epsilon>0, m$ being fixed, there exists a constant $T$ independent of $n$ such that for $n-m \geqq 4$

$$
\frac{d}{d t} \chi_{m, n}^{\sharp}(t) \geqq L\left(t-\epsilon+\sum_{1}^{m} a_{k}^{-1}\right) \quad(t \geqq T) .
$$

The proof of this theorem follows the ideas introduced in $\$ 20$. We define

$$
H_{m, n, r}^{t}(t)=\frac{1}{2 \pi i} \int_{-i \infty}^{i \infty} \frac{e^{s t}}{\prod_{m+1}^{n}\left(1-\frac{s}{a_{k}+r}\right) e^{s /\left(a_{k}+r\right)}} d s .
$$

We may show exactly as in Lemma $20 \mathrm{c}$ that

$$
e^{r t-\chi_{m, n}^{\#}(t)}=\left[\prod_{m+1}^{n}\left(1+\frac{r}{a_{k}}\right) e^{-r / a_{k}}\right]^{-1} H_{m, n, r}^{*}\left(t-\sum_{m+1}^{n} \frac{r}{a_{k}\left(a_{k}+r\right)}\right) .
$$


Let $\zeta_{m, n, r}$ be the one change of sign of $d H_{m, n, r}^{\#}(t) / d t$. It is not necessary to show, although it is true, that $\zeta_{m, n, r}$ is a continuous function of $r$. If we differentiate equation (3) once with respect to $t$ and set

$$
t=\sum_{m+1}^{n} \frac{r}{a_{k}\left(a_{k}+r\right)}+\zeta_{m, n, r}
$$

we obtain

$$
\left\{r-\frac{d}{d t} \chi_{m, n}^{\dagger}(t)\right\} e^{r t-x_{m, n}^{*}(t)}=0
$$

or

$$
\left(\frac{d}{d t}\right) \chi_{m, n}^{\#}\left(\sum_{m+1}^{n} \frac{r}{a_{k}\left(a_{k}+r\right)}+\zeta_{m, n, r}\right)=r \quad(r \geqq 0) .
$$

Let

$$
\zeta_{r}=8 \sum_{m+1}^{\infty}\left(a_{k}+r\right)^{-2} .
$$

Then by Theorem $13 \mathrm{~b},\left|\zeta_{m, n, r}\right| \leqq \zeta_{r}$. Further let

$$
\eta_{r}=\sum_{1}^{m}\left[\frac{1}{a_{k}}-\frac{r}{a_{k}\left(a_{k}+r\right)}\right] \text {. }
$$

If we set $r=L\left(t+\sum_{1}^{m} 1 / a_{k}\right)$, that is, $\sum_{1}^{\infty} r / a_{k}\left(a_{k}+r\right)-\sum_{1}^{m} 1 / a_{k}=t$, then using equation (4) and Theorem IV we have

$$
\begin{aligned}
\left(\frac{d}{d t}\right) \chi_{m, n}^{\ddagger}\left(\sum_{1}^{\infty} \frac{r}{r\left(a_{k}+r\right)}-\sum_{1}^{m} \frac{1}{a_{k}}+\eta_{r}+\zeta_{r}\right) & \\
& \geqq \frac{d}{d t} \chi_{m, n}^{\#}\left(\sum_{m+1}^{n} \frac{r}{a_{k}\left(a_{k}+r\right)}+\zeta_{m, n, r}\right),
\end{aligned}
$$

or

$$
\left(\frac{d}{d t}\right) \chi_{m, n}^{\dagger}\left(t+\eta_{r(t)}+\zeta_{r(t)}\right) \geqq L\left(t+\sum_{1}^{m} 1 / a_{k}\right) .
$$

Since $r(t)$ approaches infinity with $t$ and since $\eta_{r}=o(1), \zeta_{r}=o(1)$ as $r \rightarrow \infty$, we have, using Theorem IV again, the desired result.

By repeating the procedure used to establish Theorem $20 \mathrm{~h}$ we could avoid appealing to Schoenberg's work, at, however, the expense of considerable added complication in detail.

We shall need the following result, which is not substantially different from 
Theorem III.

THEOREM V. If the functions $\beta_{n}(u)(n=1,2, \cdots)$ are of uniformly bounded variation for $a \leqq u \leqq b$, if

$$
\lim _{n \rightarrow \infty} \beta_{n}(u)=\beta(u) \quad(a \leqq u \leqq b),
$$

and if the functions $\phi_{n}(u)(n=1,2, \cdots)$ are continuous and converging uniformly on the closed interval $a \leqq u \leqq b$ to the limit function $\phi(u)$, then

$$
\lim _{n \rightarrow \infty} \int_{a}^{b} \phi_{n}(u) d \beta_{n}(u)=\int_{a}^{b} \phi(u) d \beta(u) .
$$

ThEOREM 37b. Let $G(t) \in$ class II. Necessary and sufficient conditions that a function $f(x)$ defined for $\left(\gamma_{c}<x<\infty\right)$ be representable in the form

$$
f(x)=\int_{-\infty}^{\infty} G(x-t) d \beta(t)
$$

with $\beta(t) \in \uparrow$ are
A. $f(x) \in C^{\infty}$ $\left(\gamma_{c}<x<\infty\right)$,
B. $f(x)=o\left(e^{\alpha_{2} x}\right)$
$(x \rightarrow+\infty)$,
C. $\quad \prod_{1}^{n}\left(1-\frac{D}{a_{k}}\right) f(x) \geqq 0$
$\left(\gamma_{c}<x<\infty ; n=0,1,2, \cdots\right)$.

The necessity of conditions $A$ and $B$ is evident; that of condition $C$ follows from the equation

$$
\prod_{1}^{n}\left(1-\frac{D}{a_{k}}\right) f(x)=\int_{-\infty}^{\infty} G_{n}^{\#}\left(x+\sum_{1}^{n} \frac{1}{a_{k}}-t\right) d \beta(t) \quad\left(x>\gamma_{c}\right) .
$$

In order to establish the sufficiency of our conditions we note that from assumptions $\mathrm{A}$ and $\mathrm{B}$ and Theorem $31 \mathrm{~b}$ it follows that

$$
f^{(n)}(x)=o\left(e^{\alpha_{2} x}\right) \quad(x \rightarrow+\infty ; n=0,1,2, \cdots) .
$$

Let us set

$$
\beta_{n}(u)=\int_{0}^{u} P_{n}^{*}(D) f(t) d t \quad(n=0,1,2, \cdots) .
$$

The function $\beta_{n}(u)$ is defined for $u>\gamma_{c}-\sum_{1}^{n} 1 / a_{k}$. Using equation (5) and making succesive integrations by parts we may show just as in the proof of Theorem $29 \mathrm{~b}$ that

$$
P_{\nu_{1}}^{\#}(D) f(x)=\int_{-\infty}^{\infty} G_{\nu_{1}, n}^{\#}(x-t) d \beta_{n}(t) \quad\left(x>\gamma_{c}-\sum_{1}^{\nu_{1}} 1 / a_{k}\right)
$$




$$
P_{\nu_{2}}^{\#}(D) f(x)=\int_{-\infty}^{\infty} G_{\nu_{2}, n}^{\#}(x-t) d \beta_{n}(t) \quad\left(x>\gamma_{c}-\sum_{1}^{\nu_{2}} 1 / a_{k}\right) .
$$

Let $\eta$ be an arbitrary positive number and set $\lambda=\gamma_{c}-\sum_{1}^{\nu_{1}} 1 / a_{k}+\eta$. We define

$$
\frac{1}{A}=\inf _{n>\nu_{1}} \min _{-\infty<u<\infty}\left[\min _{t} G_{\nu_{1}, n}^{\#}(t) / G_{\nu_{1}, n}^{\mu}(\lambda-u), t \text { between } \lambda \text { and } \lambda-u\right] .
$$

It is easily verified from the shape of the curve $G_{v_{1}, n}(t)$, see $\$ 10$, that

$$
\begin{aligned}
\min _{-\infty<u<\infty}\left\{\min _{t} G_{\nu_{1}, n}^{\#}(t) / G_{\nu_{1}, n}^{\#}(\lambda-u), t\right. \text { between } & \lambda \text { and } \lambda-u\} \\
= & {\left[\underset{-\infty<t<\infty}{\max } G_{\nu_{1}, n}^{\#}(t) / G_{\nu_{1}, n}^{\#}(\lambda)\right]^{-1} . }
\end{aligned}
$$

From this and the fact that the functions $G_{\nu_{1}, n}^{A}(t)$ are converging as $n \rightarrow \infty$ to the function $G_{\nu_{1}}^{\#}(t)$ it is evident that $1 \leqq A<\infty$. By elementary estimations we obtain just as in $\$ 36$

$$
\int_{-\infty}^{\infty} G_{\nu_{1}, n}^{\#}(\lambda-t) d \beta_{n}(t) \geqq \frac{1}{A} G_{\nu_{1}, n}^{\#}(\lambda-u)\left|\int_{0}^{u} P_{n}^{\#}(D) f(t) d t\right|,
$$

that is,

$$
\left|\beta_{n}(u)\right| \leqq A\left[G_{\nu_{1}, n}^{\#}(\lambda-u)\right]^{-1} \int_{-\infty}^{\infty} G_{\nu_{1}, n}^{\#}(\lambda-t) P_{n}^{\#}(D) f(t) d t
$$

or using equation (6)

$$
\left|\beta_{n}(u)\right| \leqq\left[A P_{\nu_{1}}^{\#}(D) f(\lambda)\right]\left[G_{\nu_{1}, n}^{\#}(\lambda-u)\right]^{-1} \quad\left(u \geqq \gamma_{c}-\sum_{1}^{n} a_{k}^{-1}+\eta\right) .
$$

In exactly the same way we can prove that

$$
\left|\beta_{n}(u)\right| \leqq\left[A^{\prime} P_{\nu_{2}}^{\#}(D) f\left(\lambda^{\prime}\right)\right]\left[G_{\nu_{2}, n}^{\#}\left(\lambda^{\prime}-u\right)\right]^{-1} \quad\left(u \geqq \gamma_{c}-\sum_{1}^{n} a_{k}^{-1}+\eta\right),
$$

where $\lambda^{\prime}=\gamma_{c}-\sum_{1}^{\nu_{2}} a_{k}^{-1}+\eta$ and $A^{\prime}$ is defined just as $A$, but with $\nu_{2}$ instead of $\nu_{1}$.

From equation (8), the convergence of the functions $G_{\nu_{1}, n}(t)$ as $n \rightarrow \infty$, and the fact that the $\beta_{n}(u)$ are nondecreasing we see that for $n$ sufficiently large the functions $\beta_{n}(u)$ will be bounded and of uniformly bounded total variation in any given finite interval. Therefore by Theorem III there is a subsequence $n_{1}, n_{2}, \cdots$ and a function $\beta(u)$ of bounded variation in every finite interval such that $\lim _{i \rightarrow \infty} \beta_{n_{i}}(u)=\beta(u)(-\infty<t<\infty)$. Since $\beta(u)$ is the limit of functions which are nondecreasing it is itself nondecreasing.

We wish to show that for fixed $x>\gamma_{c}+4 \eta-\sum_{1}^{\nu_{2}} a_{k}^{-1}$ 


$$
\lim _{i \rightarrow \infty} \int_{-\infty}^{\infty} G_{\nu_{2}, n_{i}}^{\#}(x-t) d \beta_{n_{i}}(t)=\int_{-\infty}^{\infty} G_{\nu_{2}}^{t}(x-t) d \beta(t) .
$$

We first assert that given $\delta>0$ we can find $T_{1}$ and $T_{2}$ so large that

$$
\begin{array}{ll}
\int_{T}^{\infty} G_{\nu_{2}, n_{i}}^{\#} d \beta_{n_{i}}(t) \leqq \delta & \left(T \geqq T_{1} ; i=1,2, \cdots\right), \\
\int_{-\infty}^{-T} G_{\nu_{2}, n_{i}}^{\#} d \beta_{n_{i}}(t) \leqq \delta & \left(T \geqq T_{2} ; i=1,2, \cdots\right) .
\end{array}
$$

In order to avoid integrations by parts in estimating integrals of the form

$$
\int_{T}^{\infty} \phi(t) d \alpha(t)
$$

we shall use the following inequality. Let $A_{\eta}(t)$ be not less than the variation of $\alpha(u)$ for $(t-\eta \leqq u \leqq t+\eta)$ and let $\Phi_{\eta}(t)$ be not less than $\max |\phi(u)|$ for $(t-\eta \leqq u \leqq t+\eta)$. We have

$$
\left|\int_{T}^{\infty} \phi(t) d \alpha(t)\right| \leqq \frac{1}{\eta} \int_{T}^{\infty} \Phi_{\eta}(t) A_{\eta}(t) d t .
$$

This may be proved by writing

$$
\int_{T}^{\infty} \phi(t) d \alpha(t)=\sum_{n=0}^{\infty} \int_{I_{n}} \phi(t) d \alpha(t)
$$

where $I_{n}$ is the interval $(T+n \eta \leqq t \leqq T+(n+1) \eta)$. Now

$$
\left|\int_{I_{n}} \phi(t) d \alpha(t)\right| \leqq\left[\max _{t \in I_{n}}|\phi(t)|\right]\left[\operatorname{Var}_{t \in I_{n}} \alpha(t)\right] .
$$

It is clear that

$$
\max _{t \in I_{n}}|\phi(t)| \leqq \min _{t \in I_{n}} \Phi_{\eta}(t), \quad \operatorname{Var}_{t \in I_{n}} \alpha(t) \leqq \min _{t \in I_{n}} A_{\eta}(t) .
$$

Hence

$$
\left|\int_{I_{n}} \phi(t) d \alpha(t)\right| \leqq \frac{1}{\eta} \int_{I_{n}} \Phi_{\eta}(t) A_{\eta}(t) d t .
$$

Summing from $n=0$ to $n=\infty$ we obtain the desired inequality.

We have

$$
G_{\nu_{1}, n}^{*}(t)=\frac{1}{2 \pi i} \int_{-i \infty}^{i \infty} \frac{e^{s t}}{\prod_{p_{1}+1}^{n}\left(1-\frac{s}{a_{k}}\right) e^{s / a_{k}}} d s .
$$


Proceeding just as in $\$ 9$ we may show that

$$
\begin{aligned}
G_{\eta_{1}, n}^{*}(t)= & \alpha_{2}\left[\prod_{\eta_{1}+1, a_{k \neq \alpha_{2}}}^{n}\left(1-\frac{\alpha_{2}}{a_{k}}\right) e^{\alpha_{2} / a_{k}}\right] e^{\alpha_{2} t} \\
& +\frac{1}{2 \pi i} \int_{\alpha_{2}+\epsilon-i \infty}^{\alpha_{2}+\epsilon+i \infty} \frac{e^{s t}}{\prod_{\eta_{1}+1}^{n}\left(1-\frac{s}{a_{k}}\right) e^{s / a_{k}}} d s,
\end{aligned}
$$

where $\epsilon$ is a positive number such that $\alpha_{2}+\epsilon<\min _{a_{k}>a_{2}} a_{k}$. It follows easily that there is a constant $C_{1}$ such that

$$
G_{\nu_{1}, n}^{*}(t) \leqq C_{1} e^{\alpha_{2} t} \quad\left(t \leqq 0, n=\nu_{1}+1, \nu_{1}+2, \cdots\right) .
$$

Consequently we see from equation (8) that there is a constant $C_{2}$ such that

$$
\left|\beta_{n}(u)\right| \leqq C_{2} e^{\alpha_{2} u} \quad\left(u \geqq 0, n=\nu_{1}+1, \nu_{1}+2, \cdots\right) .
$$

A similar argument will show that there is a constant $C_{1}^{\prime}$ such that

$$
G_{\nu_{2}, n}^{t^{\prime}}(t) \leqq C_{1}{ }^{\prime} e^{\left(\alpha_{2}+\epsilon\right) t} \quad\left(t \leqq 0 ; n=\nu_{2}, \nu_{2}+1, \cdots\right) .
$$

From equations (8), (11), and (12) we have

$$
\begin{array}{r}
{\left[\operatorname{Var}_{t-\eta \leqq u \leqq t+n} \beta_{n_{i}}(u)\right] \leqq C_{2} e^{\alpha_{2}(t+\eta)}(u \geqq 0)} \\
{\left[\max _{t-\eta \leqq u \leqq t+\eta} G_{\nu_{2}, n_{i}}(x-u)\right] \leqq C_{1}^{\prime} e^{\left(\alpha_{2}+\epsilon\right)(x+\eta-t)}(t \geqq x+\eta) .}
\end{array}
$$

By inequality (10)

$$
\int_{T}^{\infty} G_{\nu_{2}, n_{i}}^{\#}(x-t) d \beta_{n_{i}}(t) \leqq C_{1}^{\prime} C_{2} e^{\left(\alpha_{2}+\epsilon\right)(x+\eta)} \eta^{-1} \int_{T}^{\infty} e^{\alpha_{2} t-\alpha_{2} t-\epsilon t} d t .
$$

It follows that we may choose $T_{1}$ so large that if $T \geqq T_{1}$ then

$$
\int_{T}^{\infty} G_{v_{1}, n_{i}}^{\#}(x-t) d \beta_{n_{i}}(t) \leqq \delta \quad(i=1,2, \cdots) .
$$

Similarly from equation (9) we see that there is a constant $C_{3}$ such that

$$
\begin{aligned}
\int_{-\infty}^{-T} G_{\nu_{2}, n_{i}}^{*}(x-t) d \beta_{n_{i}}(t) & \\
& \leqq C_{3} \eta^{-1} \int_{-\infty}^{-T} \exp \left[\chi_{\nu_{2}, n_{i}}^{*}\left(\lambda^{\prime}+\eta-t\right)-\chi_{\nu_{2}, n_{i}}^{*}(x-\eta-t)\right] d t .
\end{aligned}
$$

Using the mean value theorem we then have 


$$
\begin{aligned}
& \int_{-\infty}^{T} G_{\nu_{2}, n_{i}}^{\#}(x-t) d \beta_{n_{i}}(t) \\
& \quad \leqq C_{3} \eta^{-1} \int_{-\infty}^{-T} \exp \left\{-\left(x-\lambda^{\prime}-2 \eta\right) \chi_{\nu_{2}, n_{i}}^{\# \prime}[\lambda+\eta-t+\theta(x-\lambda-2 \eta)]\right\} d t,
\end{aligned}
$$

where $0<\theta<1$ and $\theta$ is a function of $t$. By Theorem IV

$$
\int_{-\infty}^{-T} G_{\nu_{2}, n_{i}}^{\#}(x-t) d \beta_{n_{i}}(t) \leqq C_{3} \eta^{-1} \int_{-\infty}^{-T} \exp \left[-\eta \chi_{\nu_{2}, n_{i}}^{\# \prime}\left(\lambda^{\prime}+\eta-t\right)\right] d t
$$

From Theorem 37a and from the fact that $\lim _{t \rightarrow \infty} L(t) / t=\infty$ we see that we may choose $T_{2}$ so large that $T \geqq T_{2}$ implies $\int_{-\infty}^{-T} G_{\nu_{2}, n_{i}}^{\#}(x-t) d \beta_{n_{i}}(t) \leqq \delta$ $(i=1,2, \cdots)$.

For any $T$ and fixed $x$, we have by Theorem $\mathrm{V}$ that

$$
\lim _{i \rightarrow \infty} \int_{-T}^{T} G_{\nu_{2}, n_{i}}^{\#}(x-t) d \beta_{n_{i}}(t)=\int_{-T}^{T} G_{\nu_{2}}^{\#}(x-t) d \beta(t) .
$$

Hence if $T \geqq \max \left(T_{1}, T_{2}\right)$ and $x>\gamma_{c}+4 \eta-\sum_{1}^{\nu_{2}} a_{k}^{-1}$,

$$
\underset{i \rightarrow \infty}{\limsup }\left|\int_{-\infty}^{\infty} G_{\nu_{2}, n_{i}}^{\#}(x-t) d \beta_{n_{i}}(t)-\int_{-T}^{T} G_{\nu_{2}}^{\#}(x-t) d \beta(t)\right| \leqq 2 \delta .
$$

Since $\delta$ is arbitrary it follows that

$$
P_{\nu_{2}}^{\#}(D) f(x)=\int_{-\infty}^{\infty} G_{\nu_{2}}(x-t) d \beta(t) \quad\left(x>\gamma_{c}+4 \eta-\sum_{1}^{\nu_{2}} a_{k}^{-1}\right) .
$$

By conclusion B of Lemma 30

$$
f(x)=g_{1} * g_{2} * \cdots * g_{\nu_{2}} * \int_{-\infty}^{\infty} G_{\nu_{2}}^{\#}(x-t) d \beta(t) \quad\left(x>\gamma_{c}+4 \eta\right) .
$$

Since the $g_{k}$ 's and $G_{\nu_{2}}^{\#}$ are non-negative and since $\beta(t) \in \uparrow$ the order of the integrations may be inverted to give

$$
f(x)=\int_{-\infty}^{\infty} G^{\#}(x-t) d \beta(t) \quad\left(x>\gamma_{c}+4 \eta\right) .
$$

Finally since $\eta$ is arbitrary, equation (13) holds for $\left(x>\gamma_{c}\right)$.

38. Determining function nondecreasing (class III).

THEOREM 38. Let $G(t) \in$ class III. Necessary and sufficient conditions that a function $f(x)$ defined for $\left(T+b+\sum_{1}^{\infty} a_{\mathfrak{k}}^{-1}<x<\infty\right)$ be representable in the form

$$
f(x)=\int_{-\infty}^{\infty} G(x-t) d \beta(t)
$$


with $\beta(t)$ defined and nondecreasing for $t>T$ are
A. $f(x) \in C^{\infty}$
$\left(T+b+\sum_{1}^{\infty} \frac{1}{a_{k}}<x<\infty\right)$
B. $f(x)=o\left(e^{\alpha_{2} x}\right)$
$(x \rightarrow+\infty)$,
C. $\prod_{1}^{n}\left(1-\frac{D}{a_{k}}\right)$
$f(x) \geqq 0\left(T+b+\sum_{1}^{\infty} \frac{1}{a_{k}}<x<\infty ; n=0,1,2, \cdots\right)$.

The proof of this theorem is so similar to the proof of Theorem 36 that it need not be given. The difficulties that arose for class II kernels disappear here because of the trivial behavior of $G(t)$ for large positive $t$.

\section{BIBILIOGRAPHY}

1. E. J. Akutowicz, The third iterate of the Laplace transform, Thesis, Harvard University, 1947 (Unpublished).

2. L. Bieberbach, Lehrbuch der Funktionentheorie, Leipzig, 1931. 646.

3. R. P. Boas, Jr., Asymptotic relations for derivatives, Duke Math. J. vol. 3 (1937) pp. 637-

4. - Inversion of a generalized Laplace integral, Proc. Nat. Acad. Sci. U.S.A. vol. 28 (1942) pp. 21-24.

5. R. P. Boas, Jr. and D. V. Widder, The iterated Stieltjes transform, Trans. Amer. Math. Soc. vol. 45 (1939) pp. 1-72.

6. H. Cramer, Mathematical methods of statistics, Princeton, 1946.

7. I. I. Hirschman, Jr., and D. V. Widder, An inversion and representation theory for convolution transforms with tctally positive kernels, Proc. Nat. Acad. Sci. U.S.A. vol. 34 (1948) pp. 152156.

8. Harry Pollard, Note on the inversion of the Laplace integral, Duke Math. J. vol. 6 (1940) pp. $420-424$.

9. - Integral transforms, Duke Math. J. vol. 13 (1946) pp. 307-330.

10. - The inversion of ihe transforms with reiterated Stieltjes kernels, Duke Math J. vol. 14 (1947) pp. 129-142.

11. _ - The integral transforms with iterated Laplace kernels, Duke Math. J. vol. 14 (1947) pp. 659-675.

12. G. Pólya and G. Szegö, Aufgaben und Lehrsätze aus der Analysis, Berlin, 1925.

13. I. J. Schoenberg, On totally positive functions, Laplace integrals, and entire functions of the Laguerre-Pólya-Schur type, Proc. Nat. Acad. Sci. U.S.A. vol. 33 (1947) pp. 11-17.

14. D. V. Widder, Inversion of the Laplace integral and the related moment problem, Trans. Amer. Math. Soc. vol. 36 (1934) pp. 107-200.

15. - - The Stieltjes transform, Trans. Amer. Math. Soc. vol. 43 (1937) pp. 7-60.

16. - The Green's function for a differential system of infinite order, Proc. Nat. Acad.

Sci. U. S. A. vol. 26 (1940) pp. 213-215.

17. - The Laplace transform, Princeton, 1941,

18. - Inversion formulas for convolution transforms, Duke Math. J. vol. 14 (1947) pp. 217-251.

19. - The inversion of a generalized Laplace transform, Proc. Nat. Acad. Sci. U.S.A. vol. 33 (1947) pp. 295-297.

HARVARD UNIVERSITY,

Cambridge, Mass. 